\title{
First clinical experience with IVR-CT system in the emergency room: Positive impact on trauma workflow
}

\author{
Daiki Wada ${ }^{1 *}$, Yasushi Nakamori ${ }^{1}$, Kazuma Yamakawa ${ }^{2}$ and Satoshi Fujimi ${ }^{1}$
}

\begin{abstract}
Recently, computed tomography $(\mathrm{CT})$ has gained importance in the early diagnostic phase of trauma care in the emergency room. We implemented a new trauma workflow concept with $\mathrm{CT}$ in our emergency room that allows emergency therapeutic intervention without relocating the patient. Times from patient arrival to $C T$ initiation, $C T$ end, and definitive intervention were significantly shorter with our new protocol than were those with the conventional CT protocol. Our new workflow concept, which provides faster time to definitive intervention, appears to be effective.
\end{abstract}

\section{Findings}

\section{Background}

Recently, computed tomography (CT) in the emergency room has become an essential part of trauma diagnostic workups. Hilbert et al. reported that eliminating patient transfer from the emergency room to the CT scanner location is of enormous benefit [1]. We implemented a new trauma workflow concept with a sliding CT scanner system with interventional radiology features (IVR-CT). The purpose of this study was to evaluate the therapeutic value of this workflow concept in terms of workup times.

\section{Materials and methods \\ Patient population}

This historical control study was conducted from February 2010 to April 2012 in a level I trauma center in Japan. Inclusion criteria were patients with blunt trauma who were admitted directly from the incident scene and required emergency bleeding control, which was defined as any emergent thoracotomy, laparotomy, or transcatheter arterial embolization (TAE). Patients in traumatic cardiorespiratory arrest at arrival were excluded. The

\footnotetext{
* Correspondence: dk0116-w@live.jp

1 Department of Emergency and Critical Care, Osaka General Medical Center,

3-1-56 Bandai-Higashi, Sumiyoshi-ku, Osaka 558-8558, Japan

Full list of author information is available at the end of the article
}

institutional review board of Osaka General Medical Center approved the conduction of this study.

\section{Setting}

Before the introduction of IVR-CT in our trauma workflow concept, the diagnostic measures used in the early treatment phase of trauma care included conventional radiography in combination with focused assessment with sonography for trauma. After completion of radiography and sonography, patients were transferred to the CT scanner, which was located on the same floor as the emergency room.

In August 2011, a new multislice IVR-CT system (Aquilion CX, TSX-101A; Toshiba Medical Systems Corp., Tochigi, Japan) was installed in our emergency room. All life-saving procedures including airway management, damage control surgery, and TAE can be performed on the $\mathrm{CT}$ examination and intervention table (Figure 1).

\section{Data collection}

Main outcome measures were time from patient arrival to CT initiation, CT end, and to the start of emergency bleeding control procedures. To evaluate the effects of this new workflow concept, data from two groups of patients were analyzed. The IVR-CT protocol group included patients admitted between August 2011 and April 2012. The conventional CT protocol group 


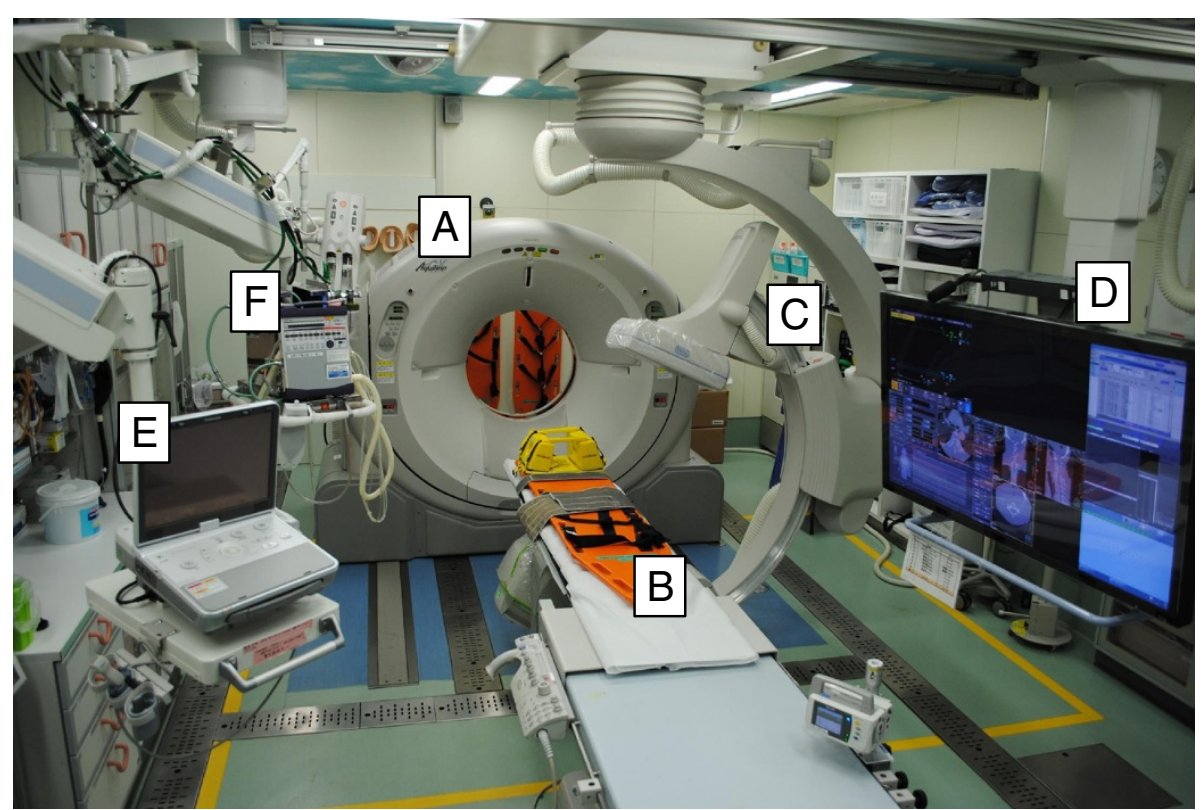

Figure 1 Photograph showing the IVR-CT system in our emergency room. All life-saving procedures including airway management, emergency surgery, and TAE can be performed on the table without relocating the patient. (A) sliding CT scanner, (B) CT examination and intervention table, (C) moveable C-arm, (D) 56-inch monitoring screen, (E) ultrasonography equipment, and (F) mechanical ventilator.

included patients admitted between February 2010 and July 2011. We also assessed 28-day mortality.

\section{Statistical analysis}

Data are expressed as group medians with interquartile ranges or numbers with percentages, as appropriate. Continuous variables were compared between groups with the Mann-Whitney $U$ test. Categorical variables were analyzed with the $\chi^{2}$ test or Fisher's exact test, as appropriate. A $P$ value of $<0.05$ was considered statistically significant.

\section{Results}

\section{Baseline characteristics}

During the study period, 48 consecutive patients fulfilled the inclusion criteria. Twenty-one patients were eligible for the IVR-CT protocol and 27 patients for the conventional CT protocol. No significant differences in baseline patient characteristics were found (Table 1). One patient in the IVR-CT protocol group and 7 patients in the conventional CT protocol group were transferred to the operating room for emergency surgery.

\section{Diagnostic and therapeutic procedures}

Time results are shown in Table 2. Compared with the conventional CT protocol, all workup times including time to the start of bleeding control procedures were significantly shorter with the IVR-CT protocol. There was no significant difference in 28 -day mortality between the two groups.

\section{Discussion}

In recent years, many major urban trauma centers have elected to install CT scanners close to or inside their emergency rooms. Although this concept substantially diminishes delays resulting from transferring patients to CT scanners, issues of patient transfer to specialized departments for definitive therapy remain as one of the rate-limiting steps in achieving maximum patient throughput. The primary advantage of our IVR-CT protocol over those of previous reports is the ability to start emergency bleeding control procedures without transferring the patient to the radiology department or operating room after completion of the diagnostic workup. Several studies previously reported that times to the start of emergency bleeding control procedures were about $80-100$ minutes [2,3], whereas those times in our trauma protocol were 45 minutes for surgery and 54 minutes for TAE, obviously shorter than those of these previous reports. However, a possible disadvantage of this workflow concept is that the emergency room can be occupied for a long period of time during bleeding control procedures.

Clarke et al. reported that delay to laparotomy in patients with intra-abdominal hemorrhage after trauma was associated with an increased risk of mortality [4]. Time to complete diagnostic tests and initiate definitive therapy, the "golden hour", is frequently mentioned in the care of seriously injured patients. Weninger et al. reported that a new management algorithm with sliding CT leads to a reduction in organ failure rates [3]. In 
Table 1 Baseline Patient Characteristics

\begin{tabular}{|c|c|c|c|}
\hline Characteristic & $\begin{array}{l}\text { IVR-CT } \\
\text { protocol } \\
(\mathrm{n}=21)\end{array}$ & $\begin{array}{c}\text { Conventional } \\
\text { CT protocol } \\
(n=27)\end{array}$ & $P$-value \\
\hline \multicolumn{4}{|l|}{ Patient characteristics } \\
\hline Age, yrs & $44(35-56)$ & $48(33-57)$ & 0.795 \\
\hline Male sex & $15(71)$ & $18(67)$ & 0.764 \\
\hline \multicolumn{4}{|l|}{ Severity of trauma } \\
\hline $\mathrm{SBP}, \mathrm{mmHg}$ & 115 (102-134) & $122(97-140)$ & 0.875 \\
\hline Heart rate, bpm & $105(80-125)$ & $104(82-130)$ & 0.976 \\
\hline RTS & $7.8(6.0-7.8)$ & $7.6(6.9-7.8)$ & 0.664 \\
\hline Hemoglobin, g/dl & $12.4(11.4-14)$ & $12.4(11.2-13.4)$ & 0.260 \\
\hline Prothrombin time, $\%$ & $54(44.7-71)$ & $51.7(46.7-71.6)$ & 0.547 \\
\hline ISS & $38(26-44)$ & $34(26-43)$ & 0.573 \\
\hline TRISS & $13.1(3.7-57.2)$ & $12.2(4.9-21.3)$ & 0.662 \\
\hline
\end{tabular}

Therapeutic interventions

Blood transfusion within $\quad 10(2-16) \quad 14(8-24) \quad 0.335$ 24 hrs, unit

Bleeding control site

$\begin{array}{cccc}\text { Chest } & 6(29) & 2(7) & 0.115 \\ \text { Abdomen } & 9(43) & 14(52) & 0.573 \\ \text { Pelvic } & 14(67) & 12(44) & 0.153\end{array}$

Bleeding control procedure

$\begin{array}{cccc}\text { TAE } & 14(67) & 2(10) & 1.000 \\ \text { Thoracotomy } & 5(24) & 18(67) & 0.574 \\ \text { Laparotomy } & 1(4) & 8(30) & 0.750\end{array}$

Data are expressed as group medians (interquartile ranges) or number (percent).SBP, systolic blood pressure; Heart rate per a minute; bpm, beat per minute; IVR, interventional radiology; CT, computed tomography; RTS, Revised Trauma Score; ISS, Injury Severity Score; TRISS, Trauma and Injury Severity Score; $\mathrm{TAE}$, transcatheter arterial embolization.

addition, Wurmb et al. reported that the rapid diagnostic workup with sliding CT might be associated with an improved outcome [5]. Thus, our IVR-CT-based workflow concept allowing faster definitive intervention may have an impact on patient survival.

\section{Table 2 Outcomes Related to Time Analysis}

\begin{tabular}{lccc}
\hline & $\begin{array}{c}\text { IVR-CT } \\
\text { protocol } \\
\text { (n=21) }\end{array}$ & $\begin{array}{c}\text { Conventional } \\
\text { CT protocol } \\
\text { (n= 27) }\end{array}$ & P-value \\
\hline Time to CT initiation, min & $10(5-17)$ & $29(25-39)$ & $<0.001$ \\
Time to CT end, min & $16(12-23)$ & $38(33-48)$ & $<0.001$ \\
Time to definitive therapy, min & & & \\
Thoracotomy/laparotomy & $45(37-65)$ & $108(81-129)$ & 0.004 \\
Transcatheter arterial embolization $^{\mathrm{b}}$ & $54(42-66)$ & $75(58-105)$ & 0.007 \\
28-day mortality, \% & $5(24)$ & $5(19)$ & 0.729 \\
\hline
\end{tabular}

Data are expressed as group medians (interquartile ranges) or number

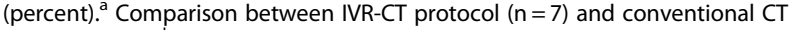
protocol $(n=9){ }^{\text {b }}$ Comparison between IVR-CT protocol $(n=14)$ and conventional CT protocol $(n=18)$.

IVR, interventional radiology; $\mathrm{CT}$, computed tomography.
We acknowledge several limitations in this study. First, this is a retrospective historical control study and not a randomized control study. Second, this study was conducted in a single institution. Third, the sample size was small. Thus, further study is required to evaluate the value of this workflow concept, including adverse events such as wound infection or sepsis that could be related to the performance of surgical procedures in the emergency room.

\section{Conclusion}

Our new trauma workflow concept using an IVR-CT system in the emergency room can facilitate definitive interventions more quickly in comparison with a conventional $\mathrm{CT}$ protocol.

\section{Abbreviations}

CT: computed tomography; IVR: interventional radiology; TAE: transcatheter arterial embolization.

\section{Authors' contributions}

DW participated in study design and in data collection and interpretation and drafted the manuscript. YN conceived the study and its design and helped to draft the manuscript. KY had a major impact on the interpretation of data and critical appraisal of the manuscript. SF participated in data interpretation. All authors read and approved the final manuscript.

\section{Competing interests}

The authors declare that they have no competing interests.

\section{Acknowledgements}

This study was supported by a Grant-in-aid from the National Mutual Insurance Federation of Agricultural Cooperatives.

\section{Author details}

'Department of Emergency and Critical Care, Osaka General Medical Center, 3-1-56 Bandai-Higashi, Sumiyoshi-ku, Osaka 558-8558, Japan. ²Department of Traumatology and Acute Critical Medicine, Osaka University Graduate School of Medicine, 2-15 Yamadaoka Suita, Osaka 565-0871, Japan.

Received: 5 June 2012 Accepted: 1 August 2012

Published: 7 August 2012

\section{References}

1. Hilbert P, Zur Nieden K, Hofmann GO, Hoeller I, Koch R, Stuttmann R: New aspects in the emergency room management of critically injured patients: a multi-slice CT-oriented care algorithm. Injury 2007, 38:552-558.

2. Fung Kon Jin PH, Gostlings JC, Ponsen KJ, van Kuijk C, Hoogerwerf N, Luitse JS: Assessment of a new trauma workflow concept implementing a sliding CT scanner in the trauma room: the effect on workup times. J Trauma 2008, 64:1320-1326

3. Weninger P, Mauritz W, Fridrich P, Spitaler R, Figl M, Kern B, Hertz H: Emergency room management of patients with blunt major trauma: evaluation of the multislice computed tomography protocol exemplified by an urban trauma center. J Trauma 2007, 62:584-591.

4. Clarke JR, Trooskin SZ, Doshi PJ, Greenwald L, Mode CJ: Time to laparotomy for intra-abdominal bleeding from trauma does affect survival for delays up to 90 minutes. J Trauma 2002, 52:420-425.

5. Wurmb T, Quaisser C, Balling H, Kredel M, Muellenbach R, Kenn W, Roewer $\mathrm{N}$, Brederlau J: Whole-body multislice computed tomography (MSCT) improves trauma care in patients requiring surgery after multiple trauma. Emerg Med J 2011, 28:300-304.

doi:10.1186/1757-7241-20-52

Cite this article as: Wada et al: First clinical experience with IVR-CT system in the emergency room: Positive impact on trauma workflow. Scandinavian Journal of Trauma, Resuscitation and Emergency Medicine 2012 20:52. 